\section{Endoscopically visualized features of gastric submucosal tumors on submucosal endoscopy}

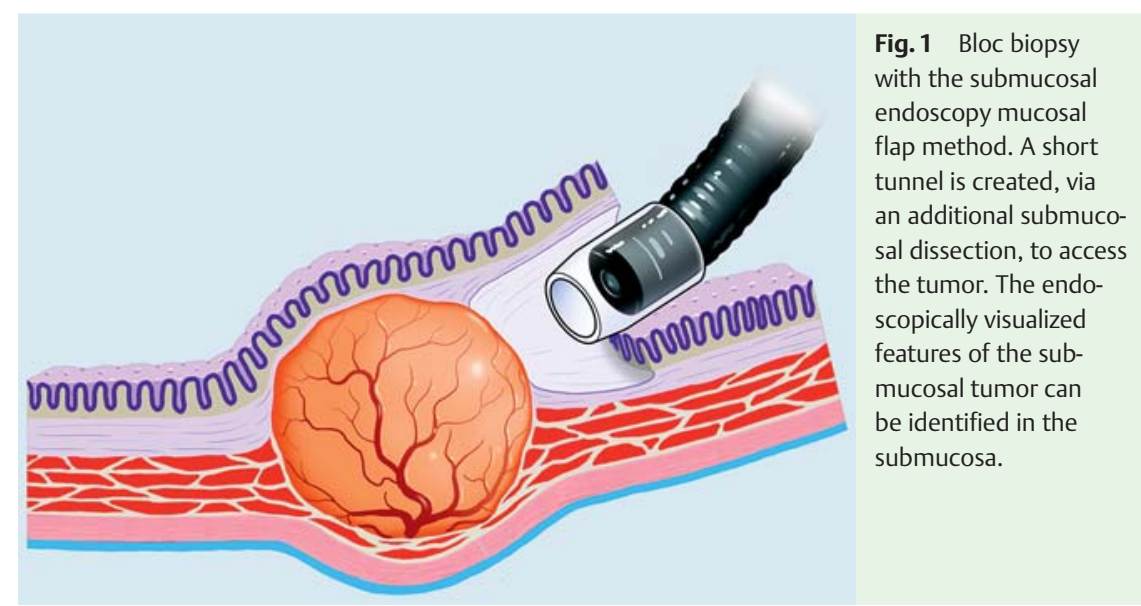

Difficulties in the preoperative diagnosis of gastrointestinal submucosal tumors by endoscopic ultrasonography and errors of tissue sampling may make invasive surgery [1] or endoscopic resection [2] inappropriate for their removal. Better methods of diagnosing submucosal tumors are therefore needed. We have found that bloc biopsy and use of the submucosal endoscopy with a mucosal flap (SEMF) method [3] under direct vision can assist in the diagnosis of submucosal tumors $[4,5]$. This method has the advantage of assessing the macroscopic characteristics of submucosal tumors. These endoscopically visualized features, identified by

\section{Video 1}

First, a bloc biopsy is obtained with the submucosal endoscopy mucosal flap method, which creates a short tunnel via an additional submucosal dissection to access the tumor. Next, the endoscopically visualized features of each submucosal tumor can be visually identified in the submucosa. The video shows representative endoscopically visualized features of different types of gastric submucosal tumors. Gastrointestinal stromal tumors are white, cloudy, round, rigid tumors; the latter feature is determined by applying pressure to the tumor with the needle-knife. Leiomyomas are white, clear, round, elastic but hard tumors. Heterotopic pancreatic tissue tumors are yellowish, multinodular, soft tumors with surface features specific to pancreatic tissue. Gastric cysts are bluish, clear, round, soft tumors with wet surfaces. Lipomas are yellow, soft tumors with adipose tissue-like characteristics. endoscopic imaging under direct view through a dissected submucosal tunnel, include the color, clarity, shape, and solidity of the tumor. Here, we describe the representative endoscopically visualized features of different types of gastric submucosal tumors.

Bloc biopsy with the SEMF method consists of five major steps: SEMF, endoscopic submucosal dissection, bloc biopsy to acquire a specimen of sufficient size, tissue collection, and clip closure of the entry point. After the creation of a tunnel into the submucosa toward the tumor, the endoscopically visualized features of a submucosal tumor can be identified in the submucosa ( $\bullet$ Fig. 1 ). The solidity of a submucosal tumor can be assessed by using a needle-knife (KD-441Q; Olympus Medical Systems Corporation, Tokyo, Japan) to apply pressure to the tumor in the area of the bloc biopsy. Different types of submucosal tumors can be classified according to four endoscopically visualized features: color, clarity, shape, and solidity. Gastrointestinal stromal tumors (GISTs) are white, cloudy, round, rigid tumors ( $\bullet$ Fig. 2); leiomyomas are white, clear, round, elastic but hard tumors ( $\bullet$ Fig. 3 ); heterotopic pancreatic tissue masses are yellowish, multinodular, soft tumors with surface features specific to pancreatic tissue ( Fig.4); gastric cysts are bluish, clear, round, soft tumors with wet surfaces ( $\bullet$ Fig.5); and lipomas are yellow, soft tumors with characteristics similar to those of adipose tissue ( $\bullet$ Fig. 6 ).

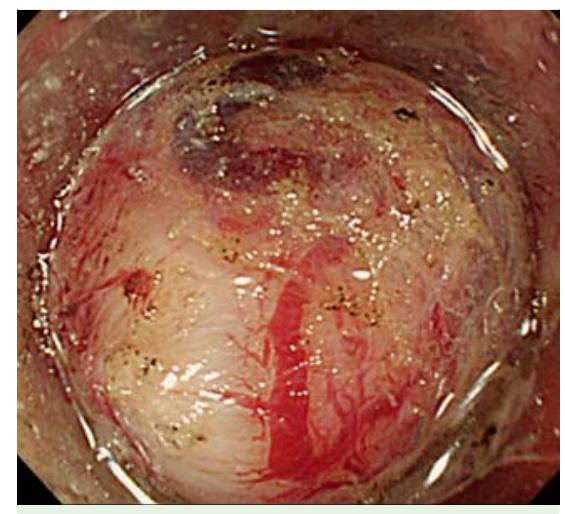

Fig. 2 Gastrointestinal stromal tumor (GIST) characteristics at submucosal endoscopy: typically white, cloudy, round, rigid tumors.

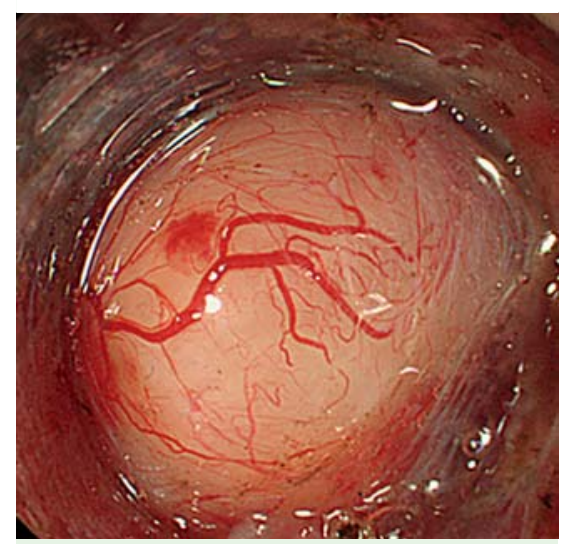

Fig. 3 Leiomyoma characteristics at submucosal endoscopy: typically white, clear, round, elastic but hard tumors.

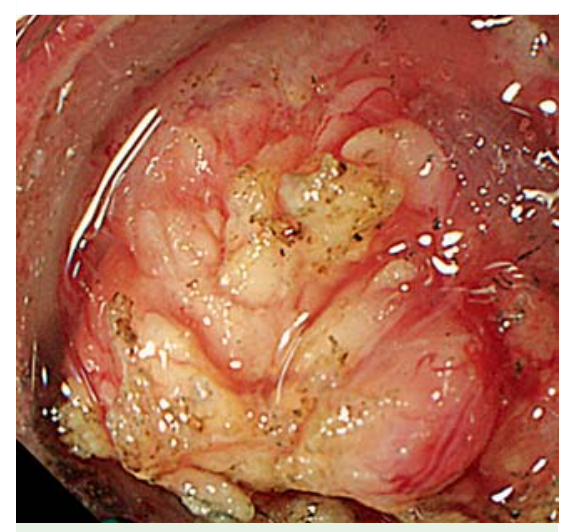

Fig.4 Heterotopic pancreatic tissue characteristics at submucosal endoscopy: typically yellowish, multinodular, soft tumors, with surface features specific to pancreatic tissue.

Submucosal tumors may be classified on the basis of features seen at submucosal endoscopy. These features may have diagnostic value that allows avoidance of unnecessary surgery ( Video 1 ). 


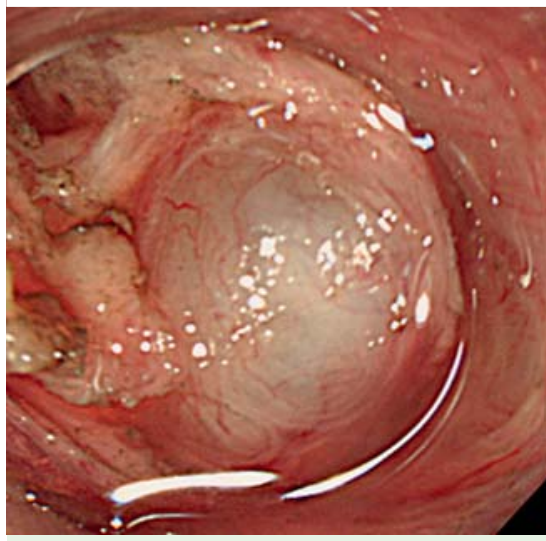

Fig. 5 Gastric cyst characteristics at submucosal endoscopy: typically bluish, clear, round, soft tumors with wet surfaces.

Endoscopy_UCTN_Code_TTT_1AO_2AC

Competing interests: None

Hideki Kobara, Hirohito Mori, Shintaro Fujihara, Noriko Nishiyama, Johji Tani, Asahiro Morishita, Makoto Oryu, Kunihiko Tsutsui, Tsutomu Masaki

Department of Gastroenterology and Neurology, Kagawa Medical University School of Medicine, Kagawa, Japan

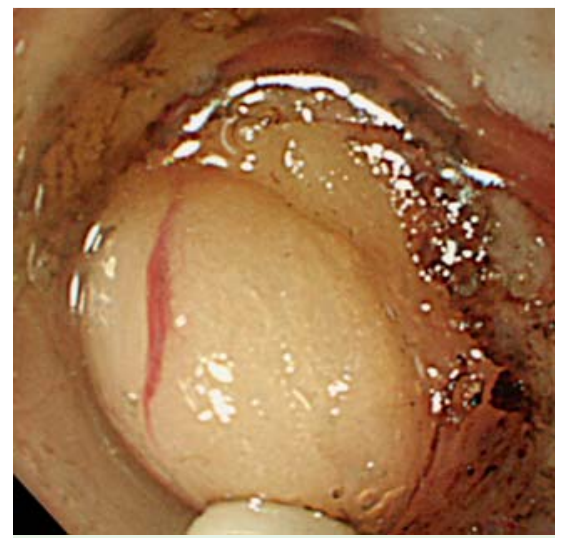

Fig. 6 Lipoma characteristics at submucosal endoscopy: typically yellow, soft tumors with adipose tissue-like characteristics.

\section{References}

1 Ormarsson OT, Gudmundsdottir I, Mårvik R. Diagnosis and treatment of gastric heterotopic pancreas. World J Surg 2006; 30: $1682-1689$

2 Inoue H, Ikeda H, Hosoya $T$ et al. Submucosal endoscopic tumor resection for subepithelial tumors in the esophagus and cardia. Endoscopy 2012; 44: 225-230

3 Sumiyama K, Gostout CJ, Rajan E et al. Submucosal endoscopy with mucosal flap safety valve. Gastrointest Endosc 2007; 65: $688-694$
4 Kobara H, Mori H, Masaki T et al. Bloc biopsy by tunneling method using the endoscopic submucosal dissection for an upper gastrointestinal submucosal tumor. Endoscopy 2012; 44: E197-E198

5 Kobara H, Mori H, Fujihara $S$ et al. Bloc biopsy by using submucosal endoscopy with a mucosal flap method for gastric subepithelial tumor tissue sampling (with video). Gastrointest Endosc 2013; 77: 141 - 145

Bibliography

DOI http://dx.doi.org/

10.1055/s-0034-1390840

Endoscopy 2014; 46: E660-E661

(c) Georg Thieme Verlag KG

Stuttgart · New York

ISSN 0013-726X

\section{Corresponding author}

Hideki Kobara, MD, PhD

Department of Gastroenterology and Neurology Kagawa Medical University School of Medicine

1750-1 Ikenobe, Miki, Kita

Kagawa 761-0793

Japan

Fax: +81-87-891-2158

kobara@med.kagawa-u.ac.jp 